\title{
Laparoscopic Surgical Algorithm to Triage the Timing of Tumor Reductive Surgery in Advanced Ovarian Cancer
}

\author{
Nicole D. Fleming, MD ${ }^{1}$, Alpa M. Nick, MD ${ }^{2,3}$, Robert L. Coleman, MD ${ }^{1}$, Shannon N. Westin, \\ MD ${ }^{1}$, Pedro T. Ramirez, MD ${ }^{1}$, Pamela T. Soliman, MD $^{1}$, Bryan Fellman ${ }^{4}$, Larissa A. Meyer, \\ MD1 ${ }^{1}$, Kathleen M. Schmeler, MD¹, Karen H. Lu, MD ${ }^{1}$, and Anil K. Sood, MD1 \\ ${ }^{1}$ Department of Gynecologic Oncology and Reproductive Sciences, The University of Texas MD \\ Anderson Cancer Center, Houston, Texas 77030 \\ ${ }^{2}$ St. Thomas Medical Partners, Gynecologic Oncology, Nashville, Tennessee 37203 \\ ${ }^{3}$ University of Tennessee Health Sciences Center, Memphis, Tennessee 38163 \\ ${ }^{4}$ Department of Biostatistics, The University of Texas MD Anderson Cancer Center, Houston, \\ Texas 77030
}

\begin{abstract}
Objective-To estimate the effects of a laparoscopic scoring algorithm to triage patients with advanced ovarian cancer to immediate or delayed debulking to improve complete gross surgical resection rates and determine the resulting clinical outcomes.
\end{abstract}

\begin{abstract}
Methods-We prospectively performed laparoscopic assessment on patients with suspected advanced stage ovarian cancer from April 2013 to December 2016 to determine primary resectability at tumor reductive surgery. Patients with medically inoperable or distant metastatic disease received neoadjuvant chemotherapy (NACT). Two-surgeon scoring was performed in a blinded fashion using a validated scoring method. Patients with predictive index value scores $<8$ were offered primary surgery and those with score $\geq 8$ received neoadjuvant chemotherapy. Univariate and multivariate analysis was performed for effects on progression-free survival (PFS).
\end{abstract}

Results-Six hundred twenty-one patients presenting with presumed advanced ovarian cancer were evaluated during the study period and 488 patients met inclusion criteria. Two hundred fifteen patients underwent laparoscopic scoring, of which 125 patients had predictive index value score $<8$ and 84 had predictive index value score $>8$. Blinded two-surgeon predictive index value scoring resulted in bivariate discordance in only $2 \%$ of cases. Tumor cytoreduction led to no gross residual disease (R0 resection) in $88 \%$ of patients in the primary surgery group and $74 \%$ in the NACT. Patients triaged to primary surgery had an improved PFS of 21.4 months compared to 12.9

Corresponding Author: Nicole D. Fleming, MD, 1155 Pressler, Unit 1362, Houston, Texas 77030, Phone: (713) 563-1792, Fax: (713)

745-1541, nfleming@mdanderson.org.

Financial Disclosure

Larissa A. Meyer has received research funding from AstraZeneca for unrelated research, and she participated in an advisory board for Clovis Oncology in October of 2016. The other authors did not report any potential conflicts of interest.

The author has indicated that he or she has met the journal's requirements for authorship.

Conflict of Interest Disclosures:

The authors have no conflicts of interest to disclose. 
months in those patients undergoing NACT $(\mathrm{p}<0.001)$. Median PFS by treatment group and residual disease status was as follows: primary surgery-R0 23.5 months; primary surgery-R1 (any gross residual disease) 17.6 months,; NACT-R0 15.5 months; NACT-R1 12.9 months ( $\mathrm{p}<0.001$ ). On multivariate analysis for PFS, baseline CA-125 ( $\mathrm{p}=0.001)$ and gross residual disease at tumor reductive surgery $(\mathrm{p}=0.01)$ were significantly associated with PFS.

Conclusion-Laparoscopic triage assessment allowed for a personalized approach to the management of advanced ovarian cancer patients and resulted in high complete surgical resection rates at tumor reductive surgery.

\section{Introduction}

Ovarian cancer is diagnosed at advanced stages in $80 \%$ of cases, leading to 5 -year survival of approximately $30 \%$. [1] Tumor reductive surgery and platinum and taxane-based chemotherapy has been the mainstay of treatment for advanced disease. [2-6] Previous studies have demonstrated a relationship between the degree of postoperative residual disease and survival. [7] Recent studies have demonstrated a clear survival benefit if resection to no gross residual disease (R0 resection) can be achieved. [8-11] More extensive surgical procedures have been performed to achieve R0 status, and have been associated with increased surgical morbidity. [11-13]

A systematic review supported the use of neoadjuvant chemotherapy (NACT) followed by interval surgery as an alternative approach in patients with high perioperative risk and low likelihood of optimal cytoreduction at primary surgery. [14] Randomized trials have compared primary cytoreduction to NACT with interval surgery and showed equivalent survival outcomes with less surgical morbidity with NACT. [15-17] Moreover, a subgroup analysis showed that patients presenting with smaller tumors experienced improved 5-year survival with primary surgery. [15]

Accurate assessment of tumor burden at initial diagnosis using pre-operative computed tomography (CT), serum CA-125, and clinical factors have been used in models with variable success and has been difficult to standardize across surgical practices. [18-21] A laparoscopy-based scoring model developed by Fagotti et al. demonstrated that a predictive index value (PIV) score of $\geq 8$ had a specificity of $100 \%$, positive predictive value of $100 \%$, and negative predictive value of $70 \%$ for predicting a suboptimal primary tumor reductive surgery. Optimal tumor reductive surgery was defined as $\leq 1 \mathrm{~cm}$ in this model. [22-24] To provide a more personalized approach to the management of advanced ovarian cancer patients at our institution, we introduced a quality improvement program to evaluate each patient, which triaged appropriate patients to laparoscopic scoring assessment using the previously validated scoring algorithm as reported by Fagotti et al. [22-24] We sought to estimate the effects of the laparoscopic scoring algorithm in patients with advanced ovarian cancer to improve complete gross surgical resection rates and determine the resulting clinical outcomes. 


\section{Materials and Methods}

This prospective quality improvement program was approved by the University of Texas MD Anderson Quality Improvement Board (QI-0139) and retrospective data review approved by the Institutional Review Board (PA16-1010). The currently ongoing program was initiated on April 1, 2013 and data analysis for primary endpoints of R0 resection rates and progression-free survival concluded December 31, 2016. All patients presenting to our institution with suspected advanced-stage ovarian cancer were prospectively screened and their data tracked. A consensus agreement among our institution's 20 gynecologic oncology faculty surgeons was achieved regarding guidelines for screening and management of these suspected advanced-stage ovarian cancer patients. Those patients with poor Eastern Cooperative Oncology Group (ECOG) scores of 3 to 4, medical co-morbidities at the time of diagnosis precluding primary surgery, newly diagnosed deep venous thrombosis or pulmonary embolus within 6 weeks of presentation, imaging confirmation of distant metastatic disease or unresectable disease were offered neoadjuvant chemotherapy. Distant metastatic and unresectable disease was defined as intraparenchymal liver or lung metastases, mediastinal adenopathy, tumor burden or adenopathy in porta hepatis region, and bulky $>2 \mathrm{~cm}$ adenopathy in the aortic and retrocaval region. Patients with suspected advanced-stage disease that were considered possible primary surgery candidates were offered laparoscopic scoring assessment to determine resectability based on the scores previously reported by Fagotti et al. (Appendix 1, available online at http://links.lww.com/ xxx) [22-24] Those patients with a predictive index value score of $<8$ were offered primary cytoreductive surgery. Those patients with a predictive index value score of $\geq 8$ received neoadjuvant chemotherapy. Patients that did not have carcinomatosis or suspected advanced stage ovarian cancer by imaging pre-operatively were not offered laparoscopic scoring assessment, however, if they were diagnosed with stage II-IV ovarian cancer based on surgical findings, they were included in the data analysis.

All patients undergoing laparoscopic scoring assessment had a blinded two-surgeon opinion on scoring to evaluate the reliability of disease assessment across different surgeons in the same clinical practice, with the availability of a third faculty surgeon if necessary to serve as a tiebreaker in cases with a discrepancy. A designated research coordinator was present at the time of diagnostic laparoscopy, collected the scoring data, and ensured all scores were collected in a blinded fashion. Laparoscopic videos were collected for each case for purposes of quality review and third faculty scoring. All videos and patient data were captured in a secure Redcap database as described below. Diagnostic laparoscopy entry technique and port placement was performed at the discretion of each faculty surgeon.

Weekly quality improvement meetings were held with a predetermined quality committee, which included reviewing all presenting patient cases, adherence to the pre-set eligibility guidelines, and reporting morbidity and outcomes. Patients undergoing primary cytoreductive surgery received adjuvant platinum-based chemotherapy for at least 6 cycles based on standard of care or on a clinical trial available at our institution during the study period. For patients undergoing NACT, patients received platinum-based chemotherapy per standard regimens or available clinical trials for a selected number of cycles per the 
discretion of the treating physician prior to interval tumor reductive surgery. Patients with progressive disease after NACT were not offered interval tumor reductive surgery.

Primary endpoints for our quality improvement project included improving complete gross resection rates at primary or interval tumor cytoreduction compared to historical data, and as a secondary endpoint, its effect on progression-free survival (PFS). Other secondary endpoints included laparoscopic scoring concordance between the two-surgeons and surgical morbidity at tumor reductive surgery. Eligibility for our survival analysis included patients with stage II to IV high-grade epithelial ovarian, fallopian tube, or primary peritoneal malignancy presenting during our study period. Patients were excluded from the analysis if they had stage I disease, low-grade epithelial malignancy, other primary malignancy, or if they presented to our institution already on NACT. Surgical outcomes were defined by the amount of residual disease at the time of tumor reductive surgery. R0 was used to define no gross residual disease and R1 if any gross residual disease was remaining $(1 \mathrm{~cm}$ or less and $>1 \mathrm{~cm}$ ) for PFS analysis. The rate of achieving R0 resection at our institution in the three years (2010-2012) prior to implementation of this quality improvement program was 54\% for primary and interval tumor reductive surgeries combined. [25]

Summary statistics such as means, standard deviations, ranges, frequencies and percentages were used to describe the study population by treatment type and compared using Chisquared, Fisher's exact, ANOVAs, or Kruskal-Wallis tests depending on the underlying distribution of the data. PFS was estimated using Kaplan and Meier product-limit estimator. PFS was measured from the date of either start of neoadjuvant chemotherapy or primary tumor reductive surgery to the date of last clinic visit, date of first recurrence or progression, or date of death. Patients who were progression free and alive were censored at date of last clinic visit. We calculated the percentage of agreement between the two surgeon scores to assess overall concordance of scores. We calculated 1) exact agreement defined as the same scores for both surgeons based on the predictive index value raw score ranging from 0-14 and 2) qualitative agreement defined as the same scores for both surgeons based on the cutoff of predictive index value $<8$ or predictive index value $\geq 8$. Stata v14.2 (College Station, TX) was used to perform all statistical analysis. Study data were collected and managed using REDCap (Research Electronic Data Capture) electronic data capture tools hosted at MD Anderson. [26] REDCap is a secure, web-based application with controlled access designed to support data capture for research studies, providing: 1) an intuitive interface for validated data entry; 2) audit trails for tracking data manipulation and export procedures; 3) automated export procedures for seamless downloads to common statistical packages; and 4) procedures for importing data from external sources.

\section{Results}

Six hundred twenty-one patients with suspected advanced-stage ovarian, fallopian tube, or primary peritoneal cancer presented to our institution during the designated study period, of which 488 patients met the inclusion criteria for analysis. Our consort diagram is detailed in Figure 1. Two-hundred seventy-three patients did not have laparoscopic scoring assessment. Patients were deemed to be medically inoperable due to diagnosis of recent pulmonary embolus or deep venous thrombosis $(n=18)$, poor ECOG performance status $(n=19)$, medical 
comorbidities limiting a primary surgery $(n=16)$, or unknown $(n=8)$. Patients were determined to have surgically unresectable disease by one of the following criteria: parenchymal liver metastases $(n=45)$, parenchymal lung metastases or pleural disease $(n=38)$, bulky lymphadenopathy $(n=46)$, or other reasons $(n=38)$.

Two hundred fifteen patients underwent laparoscopic scoring assessment, of whom 125 had a predictive index value score $<8$ and 84 had a predictive index value score $\geq 8$. Surgical data on all patients who underwent laparoscopy is summarized in Appendix 2, available online at http://links.lww.com/xxx. Six patients (3\%) were not evaluable for a predictive index value score either due to a laparoscopic trocar complication $(\mathrm{n}=5)$ or large $(>2 \mathrm{~cm})$ parenchymal liver metastases noted at the time of laparoscopy $(\mathrm{n}=1)$ and were excluded from the primary analysis. The 5 laparoscopic trocar complications $(2 \%)$ were all gastrointestinal tract injuries upon trocar entry into the abdomen. Of the 125 patients with a PIV score $<8,111$ patients went to primary surgery. Fifty-six patients had primary surgery on the same day as laparoscopy and 54 patients had primary surgery on a separate day (median 7 days following laparoscopic assessment). There was one patient that returned home following laparoscopic assessment for primary surgery and was lost to follow up. There were also 14 patients with a PIV score $<8$ that received NACT that were excluded from the primary analysis; 6 patients declined primary surgery after review of laparoscopy findings with the patient and in 8 patients, the treating physician refused primary surgery based on the laparoscopy findings. Concordance rates between the blinded two-surgeon scoring were available for the first 200 consecutive cases. $58 \%$ of cases had an exact agreement and $94 \%$ a qualitative agreement for the predictive index value scores between the first and second surgeon. A third surgeon was called into 10 cases (5\%) and agreed with the second surgeon in 4 cases, giving a discordance rate of $2 \%$. Following this concordance analysis in the first 200 cases, we moved to single surgeon scoring of all laparoscopy cases given the low discordance rate.

Clinical and demographic data on patients undergoing laparoscopic scoring assessment is summarized in Table 1. Based on the laparoscopic assessment PIV scores, 84 patients underwent NACT and 110 patients underwent primary tumor reductive surgery. Median age in both groups was 62 years. There was no difference in BMI, race, ECOG performance status or Charlson comorbidity index between the two groups. Median CA-125 was higher upon diagnosis in the NACT group ( 637 vs. $379 \mathrm{U} / \mathrm{mL}, \mathrm{p}=0.007$ ). More patients in the primary surgery group were found to have stage II disease (13\% vs. $0 \%$ ), and more patients in the NACT group were found to have stage IV disease (17\% vs. $7 \%, \mathrm{p}<0.001)$. Surgical and chemotherapy data is summarized in Table 2. In the NACT group $(n=84), 83 \%$ of patients underwent interval tumor reductive surgery, however, $17 \%$ of patients did not undergo tumor reductive surgery either due to progressive or stable disease or deteriorating medical status. Our surgical efforts led to R0 resection in $74 \%$ of patients in the NACT group and $88 \%$ in the primary surgery group. Majority of patients had an open approach to their tumor reductive surgery in each group ( $84 \%$ and $98 \%$ ), however, more patients undergoing interval tumor reductive surgery had a laparoscopic approach (16\% vs. $2 \%$, $\mathrm{p}=0.001$ ). Median OR time (371 vs. 305 minutes, $\mathrm{p}<0.001$ ), median length of hospital stay ( 5 vs. 4 days, $\mathrm{p}=0.002$ ), and median estimated blood loss ( 500 vs. $300 \mathrm{~mL}, \mathrm{p}=0.001$ ) was greater in the primary surgery group. The median number of NACT cycles was 3 (range 1-12) and the median total number of cycles of chemotherapy was 6 cycles in both groups. 
Median follow-up was 14.1 months for those patients who underwent tumor reductive surgery, and 13.6 months for all patients. In patients undergoing laparoscopic scoring assessment, those patients triaged to primary surgery had an improved PFS of 21.4 months compared to 12.9 months in those patients undergoing NACT (Figure 2A, $\mathrm{p}<0.001$ ). Median PFS by treatment group and residual disease status is included in Figure 2B. No gross residual disease (R0) was compared to any gross residual disease (R1) because the PFS curves for patients with residual disease $<1 \mathrm{~cm}$ and $\geq 1 \mathrm{~cm}$ were similar. Median PFS was as follows: primary surgery-R0 23.5 months; primary surgery-R1 17.6 months,; NACT-R0 15.5 months; NACT-R1 12.9 months ( $\mathrm{p}<0.001)$. On multivariate analysis for PFS in those patients undergoing laparoscopic scoring assessment (Table 3), baseline CA-125 (HR=1.00, 95\% CI 1.00-1.00, $\mathrm{p}=0.001$ ) and gross residual disease at tumor reductive surgery $(\mathrm{HR}=2.28,95 \%$ CI 1.20-4.34, $\mathrm{p}=0.01)$ were significantly associated with PFS.

We also performed a subset analysis on the entire cohort presenting with advanced stage ovarian cancer to observe for differences in clinical, surgical, and survival outcomes. For this subgroup analysis patients were categorized into three groups: no laparoscopy-NACT $(\mathrm{n}=245)$; laparoscopy-NACT $(\mathrm{n}=102)$; and primary surgery with or without laparoscopy $(n=138)$. Three patients from the consort diagram (Figure 1) were lost to follow up after initial assessment and were excluded from the subgroup analysis. Twenty-six patients underwent primary surgery without laparoscopy due to the absence of carcinomatosis on pre-operative imaging $(\mathrm{n}=17)$, surgery required upfront due to impending bowel obstruction or large mass occupying entire abdomen precluding laparoscopic evaluation $(\mathrm{n}=8)$, and one patient refused laparoscopy and underwent primary surgery. Comparison of clinical, demographic, and surgical data by subgroup analysis is summarized in Appendixes 3 and 4, available online at http://links.lww.com/xxx. We observed R0 resection rates of $81 \%, 76 \%$, and $88 \%$ in patients undergoing primary surgery or interval tumor reductive surgery in each subgroup, respectively. Appendix 5, available online at http://links.lww.com/xxx, lists the surgical procedures performed at the time of tumor reductive surgery in each group. Median PFS in the entire cohort by subgroup was 21.4 months for primary surgery compared to 14.1 months for no laparoscopy-NACT and 13.1 months for laparoscopy-NACT ( $<<0.001$, Appendix 6, part 1, available online at http://links.lww.com/xxx) groups. Median PFS in the entire cohort by subgroup and residual disease status is included in Appendix 7, available online at http://links.lww.com/xxx. The PFS curves for both NACT groups were similar and thus included together. Median PFS was as follows: primary surgery-R0 23.5 months; primary surgery-R1 16.4 months; NACT-R0 15.6 months; NACT-R1 13.1 months $(\mathrm{p}<0.001)$. On multivariate analysis for PFS (Appendix 8, available online at http:// links.lww.com/xxx) in subgroup analysis, ECOG performance status (HR 1.28, 95\% CI $1.02-1.61, \mathrm{p}=0.03$ ) and gross residual disease at tumor reductive surgery (HR 2.03, 95\% CI $1.30-3.19, \mathrm{p}=0.002$ ) were significantly related to PFS.

\section{Discussion}

Laparoscopic scoring assessment allowed for a more personalized approach to the management of patients with advanced-stage ovarian cancer at our institution. It resulted in an objective triage of patients to primary cytoreduction or NACT, and improved R0 resection rates at primary and interval tumor reductive surgery. However, it is important to note, that 
only $35 \%$ of all suspected or confirmed advanced stage ovarian cancer patients presenting to our institution were candidates for the laparoscopic scoring assessment after a prospective quality review process was implemented.

Given the higher surgical complexity and morbidity that can be associated complete gross resection at primary cytoreduction [12-17], the triage algorithm as we and others [22-24,27-28] have described may select the patients that would achieve the most benefit from primary surgery and can tolerate this aggressive surgical approach. Recent data published by Rutten et al. showed that implementing laparoscopic triage assessment in advanced ovarian cancer patients resulted in a significant decrease in futile laparotomy (10\% vs. $39 \%, \mathrm{p}<0.001)$ in patients undergoing primary cytoreduction. [27] However, complete gross resection of disease was only achieved in 57\% of patients at primary surgery and 34\% of patients at interval surgery. This is in comparison to our quality improvement process and implementation of laparoscopic scoring assessment which allowed for complete gross resection rates of $74-88 \%$ in both primary and interval tumor reductive surgical cases. These improved R0 resection rates translated into PFS of 23.5 months for our primary surgery group compared to 15.5 months in the NACT group ( $<<0.001$ ). This survival advantage was also seen in data presented by Fagotti et al. showing that R0 resection at primary surgery resulted in a PFS of 25 months, which was significantly longer than patients with any residual disease at primary surgery and patients undergoing interval tumor reductive surgery. However, the authors were only able to achieve R0 resection at primary and interval tumor reductive surgeries in $62 \%$ and $58 \%$ of cases, respectively. [28] It is possible that a focused quality improvement program, such as the one we implemented, with a primary goal of R0 resection, led to a more aggressive surgical initiative to obtain $\mathrm{R} 0$ results or that the algorithm led to more appropriate selection of patients to primary and interval surgery in order to achieve the $\mathrm{R} 0$ results.

In our series, there was a laparoscopic qualitative agreement in $94 \%$ of cases and a discordance rate of $2 \%$. This adds to existing data [24] evaluating the concordance of a blinded two-surgeon scoring assessment at the time of laparoscopy in advanced ovarian cancer cases. Recent data from Petrillo et al. have demonstrated a cut-off predictive index value score of $\geq 10$ instead of $\geq 8$ to predict suboptimal tumor cytoreduction [29], however, at the time of initiation of our quality improvement project, this data was not available. Upon review of our current data, 23 patients had a predictive index value score of 8 at laparoscopic assessment. Thus, using this newer cutoff would have allowed an additional 23 patients to undergo primary tumor reductive surgery. Effects of this on PFS could depend on the R0 resection rates in this group which we will consider prospectively in future studies.

We also add to the limited literature available on laparoscopic trocar injury rates in the setting of advanced stage gynecologic malignancy. We reported a $2 \%$ complication rate upon trocar entry into the abdomen, of which all were gastrointestinal tract injuries. Four injuries occurred during direct optical entry into the abdomen at Palmer's point and one injury occurred with secondary trocar placement. Fagotti et al. reported no surgical complications with open trocar entry with 300 laparoscopic assessment procedures in advanced stage ovarian cancer patients. [28] Overall, trocar entry injury rates of either vascular or gastrointestinal in nature have ranged from $0.04 \%$ to $0.08 \%$ in laparoscopic surgical cases. 
[30] Recognizing that our injury rates were slightly higher than previous reports, we implemented a radiology review process for all patients undergoing laparoscopic scoring assessment as a part of our quality improvement initiative to identify best entry location and technique.

The strengths of our study are our prospective approach to quality improvement implementation and data collection, large patient study cohort, and a large group of gynecologic oncology surgeons using a standardized triage system for patients with advanced ovarian cancer. Additional strengths include the blinded fashion of the twosurgeon scoring system and reporting our concordance rates in a large group of gynecologic oncology surgeons. Limitations of the study include the non-randomized approach. Clinical judgment did play a role in determination of candidacy for laparoscopic triage protocol. A weekly quality review committee met to discuss all cases. However, there were 8 cases out of the 488 patients with stage II-IV disease (1.6\%) in which the treating physician disagreed with the quality review committee on candidacy for laparoscopic triage assessment. This occurred more frequently early on in the adoption of the new protocol.

In conclusion, our quality improvement initiative led to a more personalized approach to patients with advanced ovarian cancer at our institution. Introduction of laparoscopic assessment for resectability allowed for appropriate triage of patients to primary cytoreduction and improved R0 resection rates with resulting positive effects on PFS. Future directions will include evaluation of our program on overall survival, patient reported outcomes, and cost-effectiveness of this triage algorithm.

\section{Supplementary Material}

Refer to Web version on PubMed Central for supplementary material.

\section{Acknowledgments}

Supported in part by the Ovarian Cancer Moon Shot Program, National Institutes of Health through M.D. Anderson's Cancer Center Support Grant CA016672 and the SPORE in ovarian cancer (CA217685), the BlantonDavis Ovarian Cancer Research Program, the Frank McGraw Memorial Chair in Cancer Research, and the American Cancer Society Research Professor Award.

\section{References}

1. American Cancer Society. Cancer Facts \& Figures. 2017

2. Bristow RE, Tomacruz RS, Armstrong DK, Trimble EL, Montz FJ. Survival of maximal cytoreductive surgery for advanced ovarian carcinoma during the platinum era: a meta-analysis. $\mathrm{J}$ Clin Oncol. 2002; 20:1248-59. [PubMed: 11870167]

3. du Bois A, Reuss A, Pujade-Lauraine E, Harter P, Ray-Coquard I, Pfisterer J. Role of surgical outcome as prognostic factor in advanced epithelial ovarian cancer: a combined exploratory analysis of 3 prospectively randomized phase 3 multicenter trials: by the Arbeitsgemeinschaft Gynaekologische Onkologie Studiengruppe Ovarialkarzinom (AGO-OVAR) and the Groupe d'Investigateurs Nationaux Pour les Etudes des Cancers de l'Ovaire (GINECO). Cancer. 2009; 115:1234-44. [PubMed: 19189349]

4. Polterauer S, Vergote I, Concin N, et al. Prognostic value of residual tumor size in patients with epithelial ovarian cancer FIGO stages IIA-IV: analysis of the OVCAD data. Int J Gynecol Oncol. $2012 ; 22: 380-85$. 
5. van der Burg ME, van Lent M, Buyse M, et al. The effect of debulking surgery after induction chemotherapy on the prognosis in advanced epithelial ovarian cancer. Gynecological Cancer Cooperative Group of the European Organization for Research and Treatment of Cancer. N Engl J Med. 1995; 332:629-34. [PubMed: 7845426]

6. Winter WE 3rd, Maxwell GL, Tian C, et al. Tumor residual after surgical cytoreduction in prediction of clinical outcome in stage IV epithelial ovarian cancer: a Gynecologic Oncology Group Study. J Clin Oncol. 2008; 26:83-89. [PubMed: 18025437]

7. Elattar A, Bryant A, Winter-Roach BA, Hatern M, Naik R. Optimal primary surgical treatment for advanced epithelial ovarian cancer. Cochrane Database Syst Rev. 2011; 8:CD007565.

8. Eisenkop SM, Spirtos NM, Friedman RL, Lin WC, Pisani AL, Perticucci S. Relative influences of tumor volume before surgery and the cytoreductive outcome on survival for patients with advanced ovarian cancer: a prospective study. Gynecol Oncol. 2003; 90:390-6. [PubMed: 12893206]

9. Chi DS, Eisenhauer EL, Lang J, et al. What is the optimal goal of primary cytoreductive surgery for bulky stage IIIC epithelial ovarian carcinoma (EOC)? Gynecol Oncol. 2006; 103:559-64. [PubMed: 16714056]

10. Aletti GD, Dowdy SC, Gostout BS, et al. Aggressive surgical effort and improved survival in advanced-stage ovarian cancer. Obstet Gynecol. 2006; 107:77-85. [PubMed: 16394043]

11. Horowitz NS, Miller A, Rungruang B, et al. Does aggressive surgery improve outcomes? Interaction between preoperative disease burden and complex surgery in patients with advancedstage ovarian cancer: an analysis of GOG 182. J Clin Oncol. 2015; 33:937-43. [PubMed: 25667285]

12. Gerestein CG, Damhuis RA, Burger CW, Kooi GS. Postoperative mortality after primary cytoreductive surgery for advanced stage epithelial ovarian cancer: a systematic review. Gynecol Oncol. 2009; 114:523-7. [PubMed: 19344936]

13. Chi DS, Zivanovic O, Levinson KL, et al. The incidence of major complications after the performance of extensive upper abdominal surgical procedures during primary cytoreduction of advanced ovarian, tubal, and peritoneal carcinomas. Gynecol Oncol. 2010; 119:38-42. [PubMed: 20609464]

14. Wright AA, Bohlke K, Armstrong DK, et al. Neoadjuvant chemotherapy for newly diagnosed, advanced ovarian cancer: Society of Gynecologic Oncology and American Society of Clinical Oncology clinical practice guideline. J Clin Oncol. 2016; 34:3460-73. [PubMed: 27502591]

15. Vergote I, Trope CG, Amant F, et al. Neoadjuvant chemotherapy or primary surgery in stage IIIC or IV ovarian cancer. N Engl J Med. 2010; 363:943-53. [PubMed: 20818904]

16. Kehoe S, Hook J, Nankivell M, et al. Primary chemotherapy versus primary surgery for newly diagnosed advanced ovarian cancer (CHORUS): an open-label, randomised, controlled, noninferiority trial. Lancet. 2015; 386:249-57. [PubMed: 26002111]

17. Onda T, Satoh T, Saito T, et al. Comparison of treatment invasiveness between upfront debulking surgery versus interval debulking surgery following neoadjuvant chemotherapy for stage III/IV ovarian, tubal, and peritoneal cancers in a phase III randomised trial: Japan Clinical Oncology Group Study JCOG0602. Eur J Cancer. 2016; 64:22-31. [PubMed: 27323348]

18. Suidan RS, Ramirez PT, Sarasohn DM, et al. A multicenter prospective trial evaluating the ability of preoperative computed tomography scan and serum CA-125 to predict suboptimal cytoreduction at primary debulking surgery for advanced ovarian, fallopian tube, and peritoneal cancer. Gynecol Oncol. 2014; 134:455-61. [PubMed: 25019568]

19. Suidan RS, Ramirez PT, Sarasohn DM, et al. A multicenter assessment of the ability of preoperative computed tomography scan and CA-125 to predict gross residual disease at primary debulking for advanced epithelial ovarian cancer. Gynecol Oncol. 2017; 145:27-31. [PubMed: 28209497]

20. Ferrandina G, Sallustio G, Fagotti A, et al. Role of CT scan-based and clinical evaluation in the preoperative prediction of optimal cytoreduction in advanced ovarian cancer: a prospective trial. $\mathrm{Br}$ J Cancer. 2009; 101:1066-73. [PubMed: 19738608]

21. Aletti GD, Eisenhauer EL, Santillan A, et al. Identification of patient groups at highest risk from traditional approach to ovarian cancer treatment. Gynecol Oncol. 2011; 120:23-8. [PubMed: 20933255] 
22. Fagotti A, Ferrandina G, Fanfani F, et al. A laparoscopy-based score to predict surgical outcome in patients with advanced ovarian carcinoma: a pilot study. Ann Surg Oncol. 2006; 13:1156-61. [PubMed: 16791447]

23. Fagotti A, Ferrandina G, Fanfani F, et al. Prospective validation of laparoscopic predictive model for optimal cytoreduction in advanced ovarian carcinoma. Am J Obstet Gynecol. 2008; 199:642e1-6. [PubMed: 18801470]

24. Fagotti A, Vizzielli G, De Iaco P, et al. A multicentric trial (Olympia-MITO 13) on the accuracy of laparoscopy to assess peritoneal spread in ovarian cancer. Am J Obstet Gynecol. 2013; 209:462e111. [PubMed: 23891632]

25. Westin SN, Nick AM, Coleman RL, et al. Impact of a personalized surgical therapy program for advanced high grade serous ovarian cancer on surgical outcomes. Presented at 16th International Gynecologic Cancer Society Biennial Meeting. 2016

26. Harris PA, Taylor R, Thielke R, Payne J, Gonzalez N, Conde JG. Research electronic data capture (REDCap) - A metadata-driven methodology and workflow process for providing translational research informatics support. J Biomed Inform. 2009; 42:377-81. [PubMed: 18929686]

27. Rutten MJ, van Meurs HS, van de Vrie R, et al. Laparoscopy to predict the result of primary cytoreductive surgery in patients with advanced ovarian cancer: a randomized controlled trial. J Clin Oncol. 2017; 35:613-621. [PubMed: 28029317]

28. Fagotti A, Vizzielli G, Fanfani F, et al. Introduction of staging laparoscopy in the management of advanced epithelial ovarian, tubal and peritoneal cancer: impact on prognosis in a single institution experience. Gynecol Oncol. 2013; 131:341-346. [PubMed: 23938372]

29. Petrillo M, Vizzielli G, Fanfani F, et al. Definition of a dynamic laparoscopic model for the prediction of incomplete cytoreduction in advanced epithelial ovarian cancer: proof of concept. Gynecol Oncol. 2015; 139:5-9. [PubMed: 26196319]

30. Munro M. Laparoscopic access: complications, technologies, and techniques. Curr Opin Obstet Gynecol. 2002; 14:365-74. [PubMed: 12151825] 


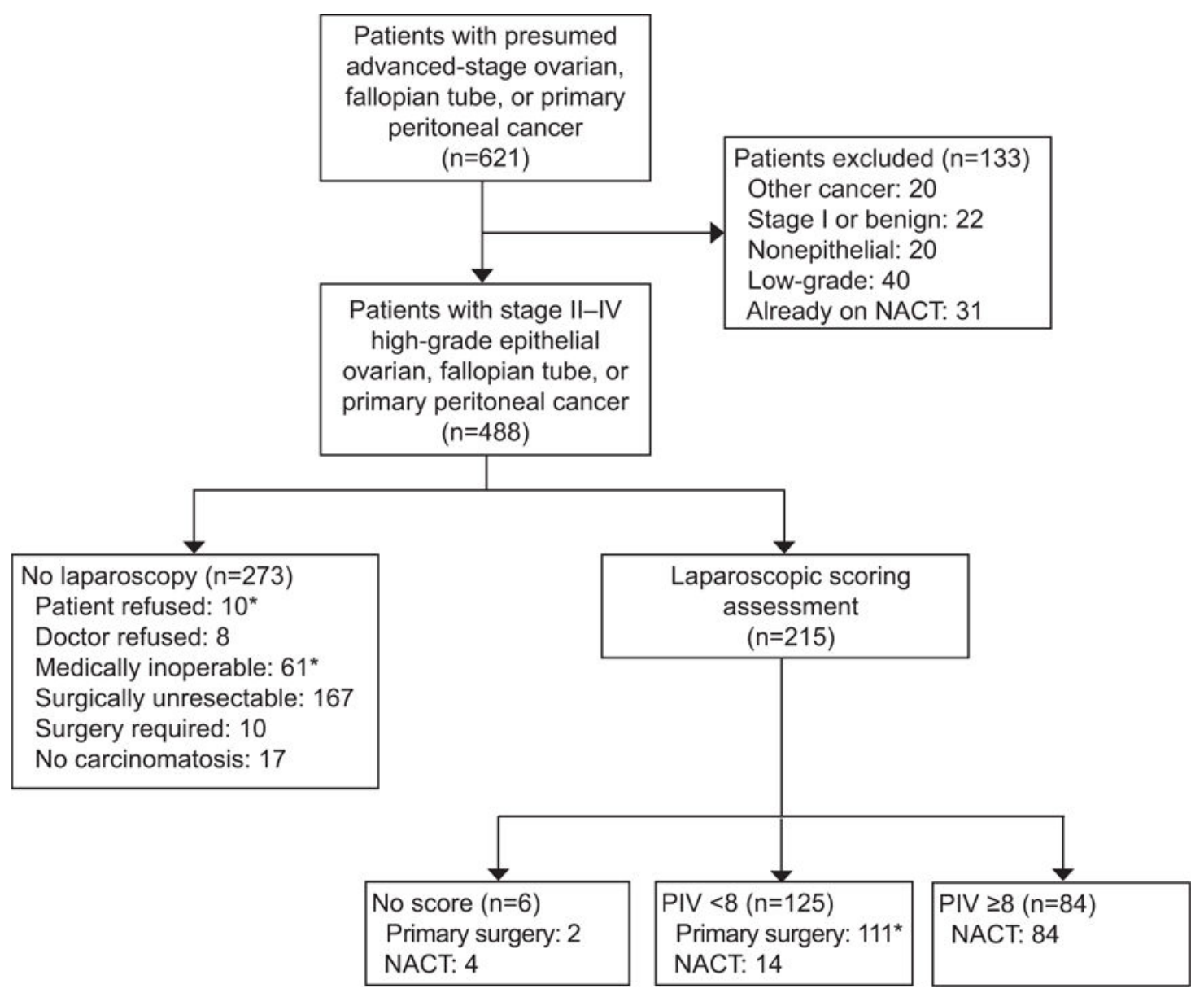

Figure 1.

Flow diagram. $* 3$ patients were lost to follow-up and not included in subgroup analysis $(\mathrm{n}=485)$. NACT, neoadjuvant chemotherapy; PIV, predictive index value. 


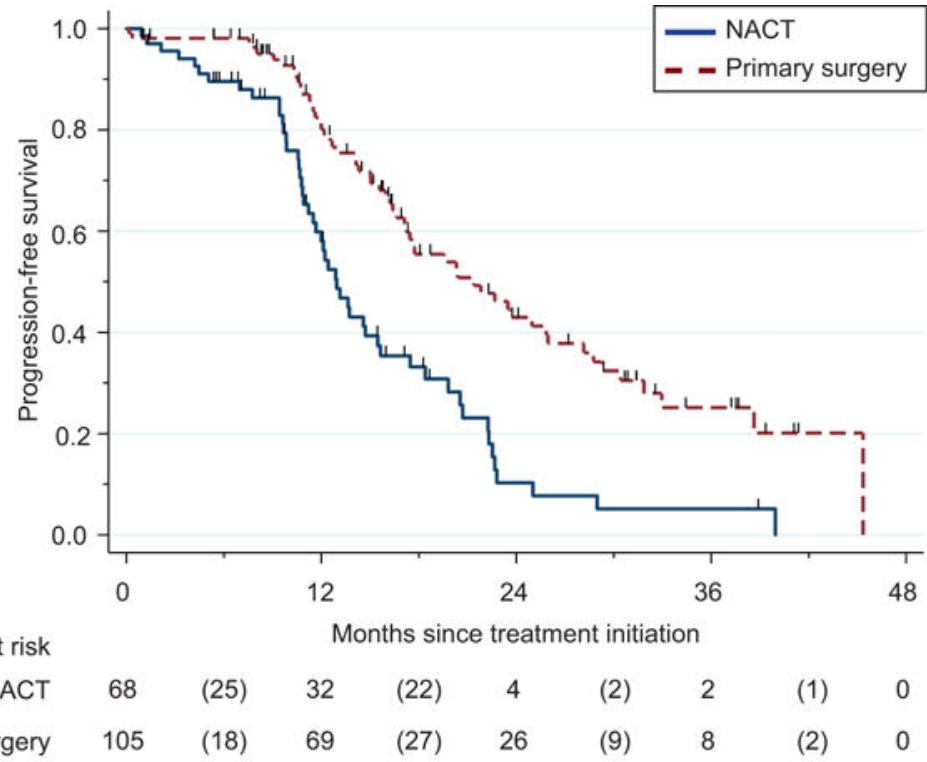

A

\begin{tabular}{rccccccccc} 
Number at risk & \multicolumn{8}{c}{ Months since treatment initiation } \\
NACT & 68 & $(25)$ & 32 & $(22)$ & 4 & $(2)$ & 2 & $(1)$ & 0 \\
Primary surgery & 105 & $(18)$ & 69 & $(27)$ & 26 & $(9)$ & 8 & $(2)$ & 0
\end{tabular}

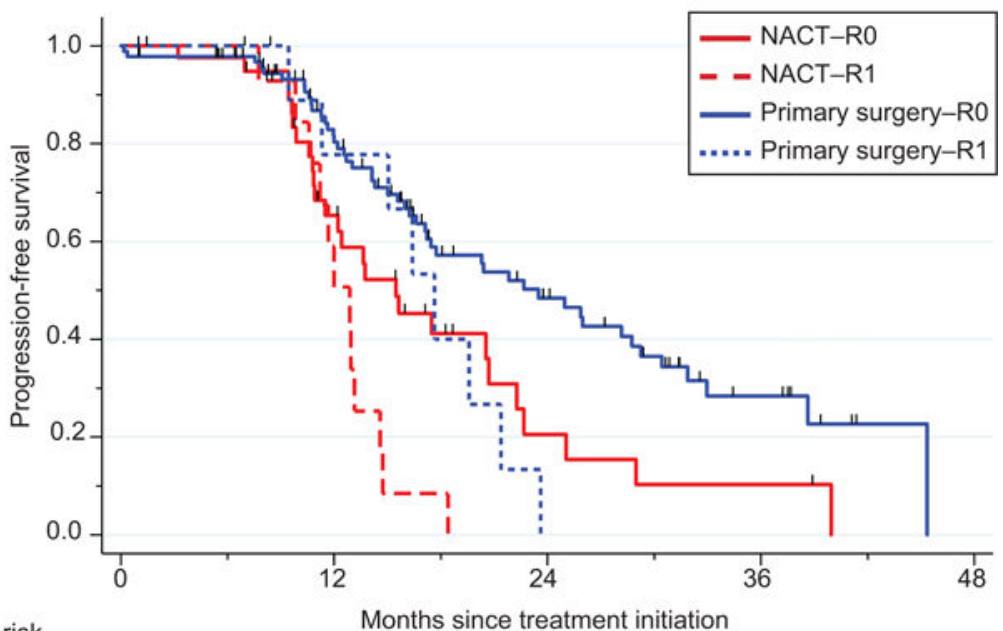

Number at risk

NACT-RO

$41 \quad$ (12) 21

(11)

4

(2)

NACT-R1 14

(6)

(6)

(0)

(1) 0

Primary surgery-R0

92

(16) 62

(21)

26

(9)

(0)

Primary surgery-R1

(2) 7

(6)

(0)

8

(2) 0

B

Figure 2.

Progression-free survival in patients undergoing laparoscopic scoring assessment by treatment group (A). Median progression-free survival was 21.4 months for primary surgery compared to 12.9 months for the NACT group $(P<.001)$. Progression-free survival in patients undergoing laparoscopic scoring assessment by residual disease and treatment group (B). Median progression-free survival was 23.5 months for primary surgery-R0, 17.6 months for primary surgery-R1, 15.5 months for NACT-R0, and 12.9 months for NACT$\mathrm{R} 1(P<.001)$. NACT, neoadjuvant chemotherapy; R0, no gross residual disease; R1, any 
gross residual disease remaining ( $\triangle \mathrm{cm}$ or $>1 \mathrm{~cm}$ ). Numbers in parentheses represent the number of events (deaths or progressions) between the two time points. 
Table 1

Clinical and demographic data for patients undergoing laparoscopic scoring assessment

\begin{tabular}{|c|c|c|c|}
\hline & $\begin{array}{l}\text { NACT } \\
(n=84)\end{array}$ & $\underset{(n=110)}{\text { Primary Surgery }}$ & p-value \\
\hline Age in years, median (range) & $62.5(36-85)$ & $62.0(37-88)$ & 0.07 \\
\hline BMI, median $\left(\mathrm{kg} / \mathrm{m}^{2}\right.$, range $)$ & $27.7(18.1-49.4)$ & $26.6(17.9-47.2)$ & 0.26 \\
\hline Race & & & 0.87 \\
\hline White & $74(91 \%)$ & $97(89 \%)$ & \\
\hline Black & $4(5 \%)$ & $8(7 \%)$ & \\
\hline Other & $3(4 \%)$ & $4(4 \%)$ & \\
\hline Missing & 3 & 1 & \\
\hline ECOG & & & 0.06 \\
\hline $0-1$ & $68(86 \%)$ & $100(95 \%)$ & \\
\hline 2 & $9(11 \%)$ & $5(5 \%)$ & \\
\hline $3-4$ & $2(3 \%)$ & $0(0 \%)$ & \\
\hline Missing & 5 & 5 & \\
\hline Charlson Comorbidity Index, median & $3(1-14)$ & $3(0-10)$ & 0.06 \\
\hline CA-125 at Dx, median (U/mL, range) & $636.7(41.5-12472)$ & $378.7(10.9-11837)$ & 0.007 \\
\hline BRCA status & & & 0.38 \\
\hline No mutation & $49(83 \%)$ & $56(70 \%)$ & \\
\hline BRCA 1 & $7(12 \%)$ & $14(18 \%)$ & \\
\hline BRCA 2 & $2(3 \%)$ & $6(8 \%)$ & \\
\hline VUS & $1(2 \%)$ & $4(5 \%)$ & \\
\hline Unknown & 25 & 30 & \\
\hline Disease site & & & $>0.99$ \\
\hline Fallopian tube & $3(4 \%)$ & $5(5 \%)$ & \\
\hline Ovarian & $72(86 \%)$ & $94(86 \%)$ & \\
\hline Primary peritoneal & $9(11 \%)$ & $11(10 \%)$ & \\
\hline Stage & & & $<0.001$ \\
\hline II & $0(0 \%)$ & $14(13 \%)$ & \\
\hline III & $70(83 \%)$ & $88(80 \%)$ & \\
\hline IVA & $6(7 \%)$ & $1(1 \%)$ & \\
\hline IVB & $8(10 \%)$ & $7(6 \%)$ & \\
\hline Histology & & & 0.06 \\
\hline Serous & $75(89 \%)$ & $88(80 \%)$ & \\
\hline Endometrioid & $0(0 \%)$ & $4(4 \%)$ & \\
\hline Clear cell & $5(6 \%)$ & $3(3 \%)$ & \\
\hline Mixed & $2(2 \%)$ & $10(9 \%)$ & \\
\hline
\end{tabular}




\begin{tabular}{|l|c|c|l|}
\hline & $\begin{array}{c}\text { NACT } \\
(\mathbf{n}=84)\end{array}$ & $\begin{array}{c}\text { Primary Surgery } \\
(\mathbf{n}=110)\end{array}$ & p-value \\
Carcinosarcoma & $2(2 \%)$ & $5(5 \%)$ & \\
\hline
\end{tabular}

Scope=Laparoscopy, NACT=Neoadjuvant chemotherapy, BMI=Body mass index, ECOG=Eastern Cooperative Oncology Group, Dx=diagnosis, VUS=Variant of uncertain significance, NOS=not otherwise specified, Adenoca=adenocarcinoma

Percentages may not equal $100 \%$ due to rounding 
Table 2

Surgical and chemotherapy data for patients undergoing laparoscopic scoring assessment

\begin{tabular}{|c|c|c|c|}
\hline & $\begin{array}{l}\text { NACT } \\
(n=84)\end{array}$ & $\underset{(n=110)}{\text { Primary Surgery }}$ & p-value \\
\hline TRS & & & $<0.001$ \\
\hline Primary & $0(0 \%)$ & $110(100 \%)$ & \\
\hline Interval & $68(83 \%)$ & $0(0 \%)$ & \\
\hline No surgery & $14(17 \%)$ & $0(0 \%)$ & \\
\hline Missing & 2 & 0 & \\
\hline Surgical approach & & & 0.001 \\
\hline Open & $57(84 \%)$ & $108(98 \%)$ & \\
\hline Laparoscopy & $11(16 \%)$ & $2(2 \%)$ & \\
\hline Robotic & $0(0 \%)$ & $0(0 \%)$ & \\
\hline Missing & 16 & 0 & \\
\hline OR time, median (minutes, range) & $305(180-688)$ & $371(16-798)$ & $<0.001$ \\
\hline LOS, median (days, range) & $4(0-43)$ & $5(0-30)$ & 0.002 \\
\hline EBL, median (mL, range) & $300(25-3300)$ & $500(50-2600)$ & 0.001 \\
\hline Residual disease at TRS & & & 0.04 \\
\hline R0 & $50(74 \%)$ & $97(88 \%)$ & \\
\hline$\leq \mathrm{cm}$ & $9(13 \%)$ & $7(6 \%)$ & \\
\hline$>1 \mathrm{~cm}$ & $9(13 \%)$ & $6(6 \%)$ & \\
\hline \multicolumn{4}{|c|}{ Number of chemotherapy cycles, median (range) } \\
\hline NACT & $3(1-12)$ & & 0.008 \\
\hline Total cycles & $6(0-12)$ & $6(0-9)$ & \\
\hline
\end{tabular}

Scope=Laparoscopy, NACT=Neoadjuvant chemotherapy, TRS=Tumor reductive surgery, LOS=Length of stay, EBL=Estimated blood loss, R0=no gross residual disease 
Table 3

Multivariate analysis for PFS in patients undergoing laparoscopic scoring assessment

\begin{tabular}{|c|c|c|}
\hline & HR $(95 \%$ CI $)$ & p-value \\
\hline Age at diagnosis & $1.02(0.98-1.06)$ & 0.39 \\
\hline ECOG status & $1.26(0.82-1.93)$ & 0.29 \\
\hline Charlson comorbidity index & $1.02(0.84-1.22)$ & 0.87 \\
\hline Baseline CA-125 & $1.00(1.00-1.00)$ & 0.001 \\
\hline Baseline platelet count & $1.00(1.00-1.00)$ & 0.18 \\
\hline \multicolumn{3}{|l|}{ Stage } \\
\hline II & - & \\
\hline III & $2.07(0.72-5.95)$ & 0.18 \\
\hline IVA & $4.31(0.92-20.18)$ & 0.06 \\
\hline IVB & $2.24(0.65-7.75)$ & 0.20 \\
\hline \multicolumn{3}{|l|}{ BRCA status } \\
\hline No mutation & - & \\
\hline BRCA $1 / 2$ & $0.66(0.32-1.36)$ & 0.26 \\
\hline Unknown & $1.04(0.59-1.82)$ & 0.91 \\
\hline \multicolumn{3}{|l|}{ Resection status } \\
\hline R0 & - & \\
\hline$\leq \mathrm{cm}$ & $2.28(1.20-4.34)$ & 0.01 \\
\hline$>1 \mathrm{~cm}$ & $2.10(0.86-5.16)$ & 0.11 \\
\hline \multicolumn{3}{|l|}{ Treatment group } \\
\hline NACT & - & \\
\hline Primary surgery & $0.69(0.42-1.14)$ & 0.14 \\
\hline
\end{tabular}

ECOG=Eastern Cooperative Oncology Group, Scope=Laparoscopy, NACT=Neoadjuvant chemotherapy 